Session 1166

\title{
A Unique, Undergraduate PLC Course
}

\author{
Michael J. Rider, Ph.D. \\ Mechanical Engineering Department \\ Ohio Northern University
}

\begin{abstract}
This paper briefly describes the PLC course (ME-429) taught at Ohio Northern University as an undergraduate technical elective. In this course the students, working in groups of two, purchase from a storeroom all Siemens /Simatic TI /Direct Logic PLC modules necessary to assemble their PLC. Each group assembles and wires their PLC under instructor supervision. During the first two weeks, they design simple ON/OFF ladder logic programs that are entered through a handheld programmer. For the remaining seven weekly projects they purchase a Dell laptop and the appropriate PLC software, DirectSOFT32, from the storeroom. Each group is responsible for installing the PLC software on their laptop, then going to the manufacturer's website and downloading the latest software updates. Ladder logic programs are designed and downloaded through the Internet to their PLC. The projects include digital logic, timers, counters, integer math, real math, PID control, and table look-ups. PLC modules used include digital I/O, analog I/O, high-speed counter, thermocouple, remote I/O, and PID control of a DC motor/tachometer arrangement. Their final weeklong project requires each group's PLC to perform a specified task along with transferring their data through an Ethernet network to each of the other PLCs in the laboratory.
\end{abstract}

This course is unique in that it requires the students to assemble, wire, and hardware debug their PLC as new modules are added throughout the quarter. At the same time, they are designing and debugging new ladder logic and stage programs on a weekly basis. The students give a short oral presentation of their weeklong project at the beginning of each laboratory period before starting the next project. This course also includes a studio classroom environment. Often after 20 or 30 minutes of lecture the students get out their laptops and program short example ladder logic exercises that reinforce the PLC concepts just discussed.

This course has been filled to capacity each quarter it has been offered. At the end of this course students have rated their PLC programming confidence and their overall satisfaction in the learning environment very high. Plans are underway to expand this course and the laboratory.

Introduction

The core engineering courses that are taught in most undergraduate programs are primarily structured around theoretical analysis and design. Often, modern control tools such as programmable logic controllers or PLCs are not covered in the curriculum especially in 
mechanical engineering. It is left for the mechanical or electrical engineering student to learn after graduation. The introduction to PLC concepts, applications, and programming places the engineering student in touch with current technology used in the manufacturing arena. PLCs are designed to operate in a manufacturing environment and interface directly with the process or the machinery being controlled. Feedback is provided by directly interfacing with different types of transducers. The purpose of the paper is to describe the way in which this topic is covered for mechanical engineering students at Ohio Northern University.

\section{History}

The classical Control Systems course (ME-419) taught during winter quarter at Ohio Northern University is a 10-week, five credit hour course. In 1994 the Mechanical Engineering Department at ONU purchased a Texas Instruments 435 PLC with two digital input and two digital output modules. Programming was done through a handheld programmer. Prior to this, the first two laboratory periods of this course taught the students about digital logic. In 1994 a third digital logic laboratory exercise using the PLC was added. The remaining seven laboratory exercises concentrated on classical control systems.

Feedback from co-op students, alumni, and employers prompted ONU to offer a technical elective in PLCs (ME-429) in the spring quarter of 1999. ME-429 was a new four credit-hour course consisting of three 50-minute lectures and a two-hour laboratory period. With the help of a grant from Marathon Oil Company, four additional PLCs with digital input and output modules and handheld programmers were purchased. In addition, analog input and output modules, and PID modules were purchased for all PLCs. IBM compatible software, called DirectSOFT, was purchased so students could design ladder logic programs on a computer, and then download their code into the PLC through its RS-232 interface. At the end of this course assessments by the students were very positive.

In the spring of 2000 Ethernet modules were purchased and the programming software was upgraded. This allowed the ladder logic programs to be downloaded at a much faster rate through Ethernet cables. The final project of the quarter required each PLC to communicate with all other PLCs on the network. Upon graduation one of the nine mechanical engineering students taking this course was offered a job because he could program a PLC and debug ladder logic programs.

In the spring of 2001 both electrical and mechanical engineering students took this technical elective course in PLCs. High-speed counter modules were purchased and added to the PLC setups. DELL laptops were purchased replacing the aging desktop computers used prior. Two of the ten students who took the course this year got a job working with PLCs after graduation.

In the spring of 2002 the programming software was upgraded again. The aging PLCs were upgraded to Direct Logic DL450 PLCs. This PLC has real number arithmetic, a real time clock, and built in PID loops.

The following year remote I/O modules were purchased for each PLC setup. In 2004 photoelectric sensors were added to each PLC setup. 


\section{Course Grading}

The course consists of nine weeklong design projects and a final exam. The grade for the course is determined by:

$\begin{array}{ll}\text { 9 Design Projects } & 60 \% \\ \text { Final Exam } & 30 \% \\ \text { Professional Conduct } & \underline{10 \%} \\ & 100 \%\end{array}$

\section{Laboratory Experience}

The design projects done by the students in the laboratory are an important part of this course. These projects reinforce the concepts covered during the lectures. Many times, lectures near the end of the quarter are cut short so that the students can program simple exercises that use the material just covered. Students work in groups of two on all projects and classroom exercises. A complete set of reference manuals from the manufacturer is located in the laboratory area for students to use, references 2 through 9 at the end of this paper.

Early laboratory design projects can be completed during the two-hour laboratory period. Later design projects require one to three additional hours outside of lab. Students use their ID cards in a swipe lock to enter the laboratory outside the allotted laboratory time. The professor is available outside of class and laboratory times to assist the students, or check their work when any wiring/rewiring is done.

\section{Course Content}

\section{Week 1}

The three lectures cover an overview of PLCs, how to assemble a DirectLogic PLC, how a PLC works, selecting a PLC, and troubleshooting a PLC. The laboratory period is spent mock-purchasing the PLC system, selecting the modules for the PLC, assembling the system, then troubleshooting it with a simple ladder logic program entered using the handheld programmer. Students mock-purchase the equipment by filling out a purchase order with current prices. The PLC and its modules are obtained from the instructor who acts as a mockvendor. Even though all PLC equipment and laptops are returned at the end of the quarter, this course forces students to write P.O.s whenever they obtain equipment for the laboratory. This allows students to get

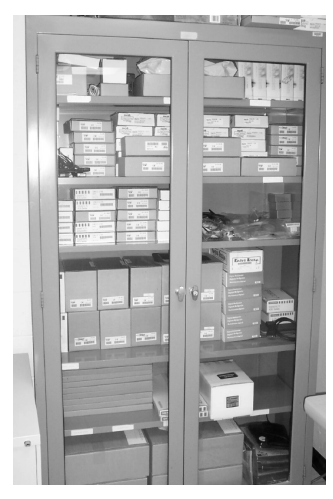
used to writing P.O.s for equipment they will need after graduation.

\section{Week 2}

The three lectures cover basic ladder logic programming, digital logic gate equivalence, and how to design simple ladder logic programs from a description of the application. In the laboratory, the students design and program, using the handheld programmer, a simple ladder logic application that forces outputs to be true/ON when certain combinations of digital inputs are set. 
Part of the design project for the week might contain the condition: Output Y1 on when inputs $\mathrm{X} 1$ and $\mathrm{X} 3$ are on, or X2 and X3 off, or X1 off and X2 on.

\section{Week 3}

The lectures during week 3 cover numbers and bases, registers and memory addressing, and how to use the Optimate OP-613 input/output interface. The Optimate OP-613 interface allows the student to program its numeric input and output range, such as: Location $1=$ input 0000 to 9999 $\mathrm{BCD}$, Location $2=$ input 20.00 to $63.00 \mathrm{BCD}$, Location $3=$ output 00.00 to $99.99 \mathrm{BCD}$ and Location $4=$ output 0000 to $9999 \mathrm{BCD}$. Input switches can also be programmed as momentary contact or toggle switches with LED indicators attached. In the laboratory students mockpurchase a laptop, load the PLC programming software, get software updates through the web from the manufacturer, then design and programming a more complicated ladder logic application.

\section{Week 4}

Timers, counters, and PLC functions involving digital bits are discussed in class of the fourth week. In the laboratory students design a traffic light with seven different preset cycle patterns. Three input switches select the appropriate cycle pattern. Students design and debug the application using the laptop and DirectSOFT32 software. The application also counts and displays the cycles completed by the traffic light so that routine maintenance can be performed after a specified number of cycles. This limit is entered through the Optimate OP-613 user interface.

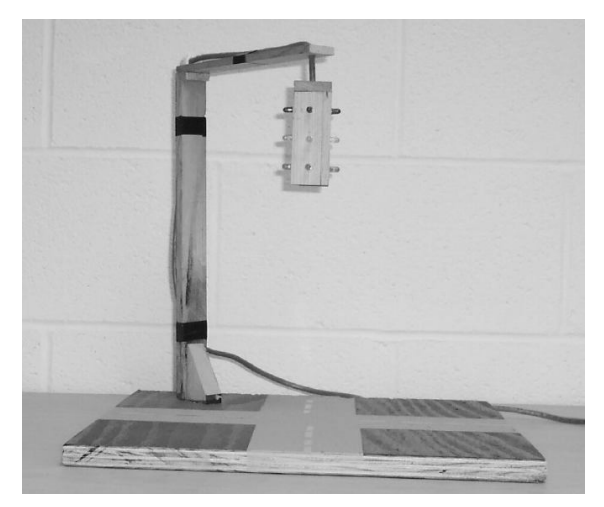

\section{Week 5}

The lectures during week five cover integer and binary coded decimal (BCD) math along with number conversion between binary, octal, hexadecimal, and BCD bases. The laboratory project typically involves an integer math game involving adding, subtracting, multiplying, and dividing at timed intervals. The objective is to start at zero and arrive at a preset value like 6773 in the shortest period of time using only add five, subtract four, multiply by two, and divide by three. If the switch to activate a math operation is left on, the operation repeats every 50 or 100 milliseconds depending upon the math operation selected. If the user goes above 9999 or below 0000 , the game terminates as a loss and the timer stops counting.

\section{Week 6}

Week six covers analog input and output modules, thermocouple input modules, and block data moves. Two laboratory projects are typically assigned during this week. The first is a thermometer, which measures temperatures using a K-type thermocouple, then displays it in both degrees Celsius and Fahrenheit. The second project is a battery tester, which can test NI-CAD batteries, dry cell 1.5-volt batteries, and dry cell 9-volt batteries. This project displays the battery voltage along with LED indicators for low voltage, normal voltage, and overcharged. 


\section{Week 7}

Week seven lectures cover data handling and table look-ups. The laboratory design project typically involves an analog input, a non-linear function table look-up, a linear interpolation, and a displayed output.

\section{Week 8}

Sequencers, PID control, and PLC networking are covered during the eighth week's lectures. The design project involves controlling a DC motor with tachometer feedback using the PLC's PID capabilities. The user can set the desired speed using a set point of the Optimate OP-613, and then observe the actual DC motor/tachometer's speed using the display feature of the user interface. A strobe-atach is used to verify the motor's actual speed and to calibrate the user interface's speed display.

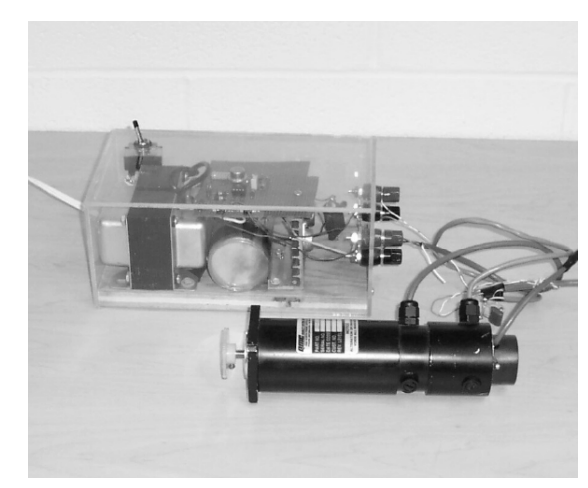

\section{Week 9}

Ladder logic jump functions, master control relay functions, stage programming, and the highspeed counter module are covered in the lecture during the ninth week. The ninth laboratory design project ties the entire quarter together. Each PLC is assigned a different task such as controlling the $\mathrm{DC}$ motor, reading the room temperature in ${ }^{\circ} \mathrm{F}$ and ${ }^{\circ} \mathrm{C}$, reading the frequency from a sine or square wave generator using the high-speed counter module, reading a battery voltage, controlling a traffic light, etc. Each PLC must network with all other PLCs in the room and share their data. For example, any PLC can change the set point for the DC motor, but only one PLC actually controls the DC motor using its PID capabilities. One PLC reads the room temperature, yet every PLC in the room must display the room temperature. One PLC reads the frequency of a sine or square wave, and every PLC in the room must display the current frequency. Only one PLC controls the traffic light, but every PLC in the room can select the light sequence, such as: flashing North-South red and flashing East-West yellow, or the standard green, yellow, red cycle.

For this project students must debug their own ladder logic program as well as the ladder logic programs of their fellow students when any part of the networking project does not work correctly. All students receive the same grade for this laboratory design project.

\section{Week 10}

Week ten's lecture continues with stage programming, remote $\mathrm{I} / \mathrm{O}$, master/slave setups, and a review of the topics covered during the quarter. Typically Monday's includes a studio classroom exercise where the students design and debug several simple stage programs. The laboratory period is used to check back in the PLC and its modules, the laptop, and all miscellaneous equipment used during the quarter. Items are reboxed for the next group of students to check out and assemble.

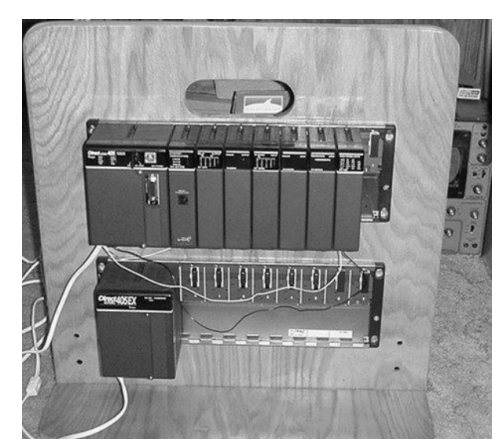


Final Examination Week

A comprehensive final examination is given during the eleventh week. Each student must demonstrate his/her knowledge of PLCs and PLC programming during the exam without the aid of his/her laboratory partner. The examination is open book, open notes. The reference manuals can be used during the exam.

Future Goals

Because of the popularity of this course, the laboratory area is being relocated to a larger room and additional PLC setups and laptops are being purchased. Currently there are no plans to change this course from a technical elective to a required mechanical engineering course.

\section{Conclusions}

Programmable logic controllers or PLCs are a very important part of factory automation. Engineers in manufacturing need to understand and be able to use PLCs in their job. Feedback from former students who took this course has been very positive. I believe all undergraduateengineering students should have the opportunity to learn about PLCs especially if they are interested in the manufacturing area.

Bibliography

[1] Rider, Michael J., "Principles of the DL405 Family Programmable Logic Controllers," ONU Publishing, $3^{\text {rd }}$ edition, 2003.

[2] AutomationDirect.com Incorporated, "DL405 PLC User Manual," $3^{\text {rd }}$ edition, Rev C, 2000.

[3] AutomationDirect.com Incorporated, "DL405 Analog User Manual,” $3^{\text {rd }}$ edition, 2000.

[4] AutomationDirect.com Incorporated, "DL405 Remote I/O User Manual," $3^{\text {rd }}$ edition, 2000.

[5] AutomationDirect.com Incorporated, "Ethernet Communications Manual," $3^{\text {rd }}$ edition, 2000.

[6] AutomationDirect.com Incorporated, "DL405 High Speed Counter Manual," $3^{\text {rd }}$ edition, 2000.

[7] AutomationDirect.com Incorporated, "Optimate OP-613 Setpoint Operator Panel User Manual," $3^{\text {rd }}$ edition, 2000 .

[8] AutomationDirect.com Incorporated, "DirectSoft4 User Manual," $3^{\text {rd }}$ edition, 2002.

[9] AutomationDirect.com Incorporated, "Part Catalog," Volume 8, 2002.

Biography

Proceedings of the 2004 American Society for Engineering Education Annual Conference \& Exposition. Copyright (C) 2004, American Society for Engineering Education 
Session 1166

DR. MICHAEL RIDER is a professor of Mechanical Engineering, and has taught at ONU for twenty-four years. His Doctorate is from Purdue University. He has taught courses in engineering drawing, statics, dynamics, advanced strength of materials, mechanisms, mechanical design of components, computer graphics, numerical methods, control systems, and Fortran programming, and PLC programming. 